OPEN ACCESS

Edited by:

Andrew Zloza,

Rush University Medical Center,

United States

Reviewed by:

Praveen Bommareddy,

Rutgers, The State University of

New Jersey, United States

Rksubbarao Malireddi,

St. Jude Children's Research Hospital,

United States

*Correspondence:

Jianmin Liang

liangjm@jlu.edu.cn

Pengfei Ge

gepf@jlu.edu.cn

Specialty section:

This article was submitted to

Cancer Molecular Targets and Therapeutics,

a section of the journal

Frontiers in Oncology

Received: 30 November 2020

Accepted: 20 January 2021

Published: 26 February 2021

Citation:

Wang L, Qin X, Liang J and Ge P (2021) Induction of Pyroptosis:

A Promising Strategy

for Cancer Treatment.

Front. Oncol. 11:635774.

doi: 10.3389/fonc.2021.635774

\section{Induction of Pyroptosis: A Promising Strategy for Cancer Treatment}

\author{
Lei Wang ${ }^{1}$, Xiaowei Qin ${ }^{1}$, Jianmin Liang ${ }^{2 *}$ and Pengfei Ge ${ }^{1 *}$ \\ 1 Department of Neurosurgery, First Hospital of Jilin University, Jilin University, Changchun, China, ${ }^{2}$ Department of Pediatric \\ Neurology, First Hospital of Jilin University, Jilin University, Changchun, China
}

Pyroptosis, a lytic pro-inflammatory type of programmed cell death, has been widely studied in diverse inflammatory disease models. Membrane perforation and cell swelling induced by cleaved gasdermin family members is the main characteristic of pyroptosis. Emerging evidence has revealed a complicated relationship between pyroptosis and cancer. On the one hand, as inflammatory cell death, pyroptosis provides a comfortable environment for tumor proliferation. On the other hand, excessive activation of pyroptosis can inhibit the development of tumor cells. In this review, we first summarized the latest progress about the molecular mechanism of pyroptosis. Then, members from gasdermin family, the central molecules of pyroptosis which formed pores on the cell membrane, were highlighted. In the second part of this review, we summarized drugs that induced pyroptosis in different tumors and their concrete mechanisms based on recent literature reports. In the final section, we discussed several hotspots in pyroptosis and cancer therapy, which will point out the direction of sequent research. In brief, inducing pyroptosis in cancer cells is a promising strategy for cancer therapy.

Keywords: pyroptosis, cancer, inflammasome, antitumor immunity, gasdermin, caspase

\section{INTRODUCTION}

The dynamic balance among cell proliferation, differentiation and death plays an important role in the physiological and pathological processes of multicellular organisms (1). Based on considerable research on cell death mechanisms, biologists have found multiple cell death forms such as apoptosis, necrosis, necroptosis, ferroptosis, etc (2). In 1999, researchers found that caspase-1 was activated in the cell death induced by Salmonella. The cell death induced by Salmonella was initially considered as caspase-1-dependent apoptosis (3). However, in Brennan and Cookson's research, Salmonella-induced cell death exhibited utterly different characteristics from apoptosis. Caspase-3 and PARP1 were not activated in Salmonella-infected macrophages. What's more, the cell membrane integrity of infected macrophages was destroyed while apoptosis cells usually had intact membranes (4). All of the differences above indicated that Salmonella-induced macrophage death was distinct from the well-known apoptosis. Thus, pyroptosis, an inflammatory programmed cell death (PCD), was proposed to elucidate such a cell death phenomenon in 2001 (5). The main characteristics of pyroptosis include membrane perforation, cell swelling, the release of cellular content, chromatin condensation and DNA fragmentation $(6,7)$. In the canonical inflammasome pathway of pyroptosis, activated caspase- 1 promotes the maturation of IL-1 $\beta$ and IL-18. Meanwhile, activated caspase- 1 also cleaves GSDMD into the C-terminal domain and $\mathrm{N}$-terminal domain. Then 
the GSDMD-NT forms pores in the plasma membrane $(6,8,9)$. In vertebrates, pyroptosis is beneficial for the clearance of pathogen and enhancing innate immunity (10). As a promising research direction, pyroptosis plays important roles in diverse diseases such as hepatitis, atherosclerosis, neurodegeneration, tumor, etc. $(1,11-13)$. Pyroptosis performs dual effects on tumors. On the one hand, pyroptosis-associated inflammatory cytokines and pathways promote tumor growth, invasion and drug resistance $(14,15)$. On the other hand, inducing pyroptosis directly suppresses tumor proliferation (16). Therefore, relevant studies on pyroptosis have positive significance for developing new tumor treatment regimens, reducing chemotherapeutic drug resistance and improving the life quality of tumor patients. In this review, we aimed to summarize and evaluate the recent studies focused on pyroptosis and cancer therapy. Subsequently, several hotspots in this field were discussed in detail. The review of earlier work could help us to define the direction of future research. We hope there will be more high-quality studies of tumor therapy based on pyroptosis in the future.

\section{SIGNAL PATHWAYS OF PYROPTOSIS}

\section{Canonical Inflammasome Pathway}

Activation of the inflammasome is the basis for caspase-1dependent pyroptosis. The inflammasome complex is usually composed of pattern recognition receptors (PRRs), inflammatory caspases and in some cases, an adapter protein that connects these proteins (17). The most common PRRs in the inflammasome complex comprise NLRP1, NLRP3, NLRC4, and AIM2 (18), which could be activated by various stimulation. For example, NLRP1 identifies the Bacillus anthracis lethal toxin to induce pyroptosis (19). NLRC4 is responded to bacterial protein, while AIM2 is mainly activated by bacteria or double-stranded DNA in cells infected by viruses. NLRP3, the best-studied PRRs, can be activated by various factors, including bacterial toxins, viral doublestranded RNA, adenosine triphosphoric acid, ROS and endogenous damage signals (10). Activated NLRP3 or AIM2 forms a macromolecule complex with adapter protein ASC and procaspase-1, further initiating pyroptosis. Besides, receptor proteins with caspase recruitment domain (CARD) such as NLRP1 or NLRC4 also recruit pro-caspase-1 and promote its proteolysis directly (15). Earlier research indicated that caspase-1 provoked the maturation of inflammatory cytokines such as IL-1 $\beta$, IL-18, although the specific mechanism of caspase-1 inducing pyroptosis was elusive. In 2015, Feng Shao and colleagues found GSDMD knockdown in iBMDM cells reversed caspase-1-dependent pyroptosis, which demonstrated the crucial role of GSDMD in pyroptosis. GSDMD, a member of gasdermin family, is highly conserved among mammalians. During the occurrence of pyroptosis, caspase-1 cleaves GSDMD into GSDMD-CT and GSDMD-NT with molecular weights of $22 \mathrm{kd}$ and $31 \mathrm{kd}$ respectively. Subsequently, due to their lipophilic property, GSDMD-NT is aggregated in the inner side of the cytomembrane to generate lots of pores, which leads to cell swelling, membrane perforation and release of cellular content such as IL-1 $\beta$ and
IL-18. Binding GSDMD-CT to GSDMD-NT inhibits all changes above $(8,9,20)$.

\section{Noncanonical Inflammasome Pathway}

The noncanonical pyroptosis pathway is independent of the inflammasome complex. Relevant studies have shown that bacterial Lipopolysaccharide (LPS) directly activated human caspase- $4 / 5$ and mouse caspase- 11 . Then the activated caspases cleaved GSDMD to perforate cell membrane $(21,22)$. In addition, although caspase-4/5/11 doesn't directly trigger hydrolysis of IL-1 $\beta$ and IL-18, the potassium efflux from ruptured cells still induces NLRP3/caspase-1 activation, ultimately leading to maturation and release of IL-1 $\beta$ and IL18. The maturation of IL-1 $\beta$ and IL-18 in the noncanonical pathway is independent of caspase-4/5/11 (20, 23, 24).

\section{Other Pathways Inducing Pyroptosis}

Apart from caspase-1/4/5/11, some non-inflammatory caspases also cause pyroptosis. GSDMD and GSDME, both of which belong to the gasdermin family, could be cleaved to generate an $\mathrm{N}$-terminal with a perforating effect. The morphology of TNF- $\alpha$-induced Hela cell death was similar to pyroptosis accompanied by activation of caspase-3/7 and GSDME. Knocking down caspase-3 switched pyroptosis to apoptosis through inactivating GSDME. Thus, researchers hypothesized that activated caspase-3, induced by TNF- $\alpha$, independently cleaved GSDME to promote pyroptosis. Subsequent studies confirmed that caspase-3 triggered GSDMEdependent pyroptosis in high GSDME expression cells in contrast to apoptosis in cells with low GSDME expression (25-27). Another non-inflammatory caspase inducing pyroptosis is caspase-8. Yersinia activates RIPK1/caspase- 8 pathway by suppressing TGF- $\beta$ activated kinase-1 (TAK1). After that, activated caspase- 8 cleaves GSDMD and GSDME to elicit pyroptosis (28-30). Besides, caspase-6 was recently found to enhance the interaction between RIPK3 and ZBP1 to activate NLRP3/caspase-1 signal pathway (31). As a newfound cell death pathway, the molecular mechanisms of pyroptosis deserve further investigation (Figure 1).

\section{GASDERMIN FAMILY PLAYS A CENTRAL ROLE IN PYROPTOSIS}

So far, researchers have classified the human gasdermin family into six categories according to their distinction of conserved sequence, including GSDMA, GSDMB, GSDMC, GSDMD, GSDME/DFNA5, and DFNB59 (32). Except for DFNB59, the rest molecules from gasdermin family are activated by cleaving into C-terminal and $\mathrm{N}$ terminal. Owing to the lipophilicity and perforating ability of the $\mathrm{N}$ terminal, the whole gasdermin family cause pyroptosis, which is inhibited by binding to the C-terminal (9). Early studies on pyroptosis mainly focused on inflammatory caspase-1/4/5/11 and GSDMD. Thus, pyroptosis was defined as inflammatory cell death. With in-depth research, scientists turned their attention to gasdermin family that directly destroyed cell membranes. Due to the pore formation property of gasdermin family, Some researchers redefined pyroptosis as gasdermin-mediated programmed cell death 


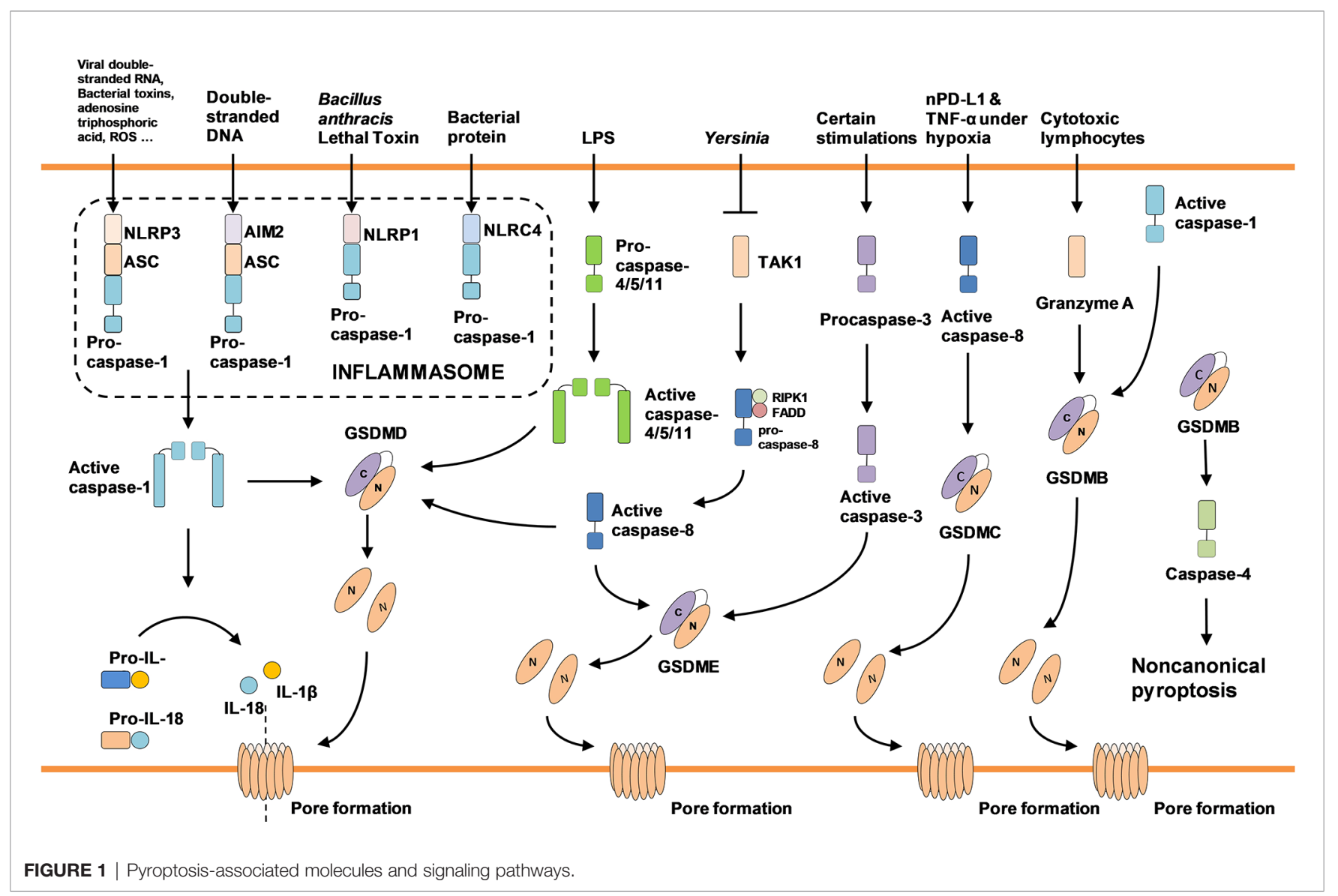

(20). Whether participation of caspase should be necessary for pyroptosis is still controversial.

GSDMD is the earliest discovered pyroptosis-associated gasdermin. In 2015, two separate teams led by Feng Shao and Vishva M Dixit demonstrated respectively that GSDMD was involved in canonical and noncanonical pyroptosis $(8,22)$. Up to now, it has been found that GSDMD was cleaved by inflammatory caspase-1/4/5/11 or non-inflammatory caspase- 8 to elicit PCD. In neutrophils, GSDMD is cleaved by neutrophil elastase at C268 (seven amino acids upstream of the caspase cleavage site) to induce pyroptosis (33). GSDME is mainly activated by noninflammatory caspase (caspase-3/8). Mutations in mice GSDMA3 exhibit hair-loss phenotypes (12). Specific GSDMA3 mutations (T278P, L343P, Y344C, A348T, and 412EA) were found to interfere with the combination of GSDMA3-CT and GSDMA3NT. Therefore, researchers speculated that the hair-loss was triggered by GSDMA3-NT-induced pyroptosis (8). Different from Feng Shao's view that caspase- 1 was unable to activate GSDMB, Panganiban found GSDMB was cleaved at the D236 position to induce pyroptosis (34). Another recent study demonstrated that GSDMB was cleaved by granzyme A released from cytotoxic lymphocytes, promoting our comprehension of cytotoxicity of lymphocytes (35). GSDMB is upregulated in patients with sepsis or Crohn's disease. Full-length GSDMB binds to caspase- 4 and activates noncanonical pyroptosis through caspase-4/GSDMD pathway. High expression of GSDMB is not directly involved in membrane perforating (36).
Similar to other gasdermins, the N-terminal of GSDMC can lead to pore formation (9). In recent research, GSDMC expression was transcriptionally activated by nPD-L1 under hypoxia. The overexpressed GSDMC was subsequently cleaved by caspase- 8 and switched TNF- $\alpha$ induced apoptosis to pyroptosis (37). Although extensive work has been finished in this field, it remains to be further explored whether there are undiscovered mechanisms for gasdermins to induce pyroptosis.

\section{SIMILARITIES, DIFFERENCES, AND CROSS-TALK AMONG PYROPTOSIS, APOPTOSIS, AND NECROPTOSIS}

Pyroptosis, apoptosis and necroptosis are three PCD pathways that have been intensively investigated recently. Although induced by distinct mechanisms, the three modes of cell death share many similarities and could be activated alone or simultaneously depending on diverse cellular contexts $(26,30,38,39)$. To better comprehend PCD in cancer treatment, it is necessary to distinguish their main characteristics briefly (Table 1). Apoptosis is one of the earliest defined PCD that widely participates in many biological processes such as embryogenesis, aging, infection and tumor regression. The major morphological characteristics of apoptosis including membrane blebbing, cell shrinkage, chromatin 
TABLE 1 | Similarities and differences among pyroptosis, apoptosis, and necroptosis.

\begin{tabular}{|c|c|c|c|}
\hline Items & Pyroptosis & Apoptosis & Necroptosis \\
\hline Morphology of cell membrane & Membrane permeabilization, cell swelling & Membrane blebbing, cell shrinkage & Membrane permeabilization, cell swelling \\
\hline Morphology of nucleus & Integral nucleus, chromatin condensation & Nuclear fragmentation, chromatin condensation & Integral nucleus \\
\hline Special constructions & Pyroptotic bodies & Apoptotic bodies & None \\
\hline Release of cellular contents & DAMPs, inflammatory cytokines & Particular DAMPs such as ATP, HMGB1 & DAMPs, inflammatory cytokines \\
\hline Key molecules & Caspase-1/4/5/11, GSDMD, GSDME & Caspase-2/3/6/7/8/9/10 & RIP1, RIP3, MLKL \\
\hline
\end{tabular}

condensation, nuclear fragmentation, and the formation of apoptotic bodies (40). Distinct from apoptotic cells that retain intact membrane, cell membrane under pyroptosis and necroptosis are perforated by gasdermins and MLKL. Therefore, cell swelling and membrane rupture are usually observed in pyroptotic and necroptotic cells $(6,41,42)$. Since the perforation triggered by GSDMD is less ion-selective than MLKL, the cell swelling is milder in pyroptosis $(43,44)$. Consistent with apoptosis, DNA fragmentation and chromatin condensation are displayed in pyroptotic cells. However, pyroptosis exhibits DNA damage at a lower intensity with an integral nucleus (6). Besides, intact nuclei are also observed in necroptotic cells (42). As a result of cell membrane damage, several damage-associated molecular patterns (DAMPs) and inflammatory factors are released from pyroptotic and necroptotic cells, which subsequently triggered a potent inflammatory response (45). Reversely, apoptosis is traditionally considered as a non-inflammatory process although the emission of particular DAMPs such as ATP and HMGB1 were demonstrated in apoptosis according to recent literature $(46,47)$. In terms of molecular mechanisms, caspases are widely involved in the three PCDs. In canonical and noncanonical pyroptosis, inflammatory caspases (e.g., caspase-1, -4, -5, -11) are generally activated to initiate the cleavage of gasdermins (18). Another group of caspases (e.g., caspase- $2,-3,-6,-7,-8,-9,-10$ ) are thought to participate in the initiation and execution of apoptotic pathways (48). Although caspases are dispensable for necroptosis, the inactivation or absence of caspase- 8 is a prerequisite for necrosome formation and necroptosis activation (45). Though pyroptosis, apoptosis and necroptosis are three distinct PCDs with various molecular mechanisms and morphological characteristics, recent reports indicated the existence of crosstalk among them. Caspase-3/8, general associated with apoptosis, were demonstrated to cleave GSDME or GSDMD and induce pyroptosis $(25,29)$. In contrast, the inflammatory caspase- 1 could activate apoptosis in the absence of GSDMD (49). Crosstalk between pyroptosis and necroptosis was also discovered in recent years. MLKL, the executioner of necroptosis, activates NLRP3 inflammasome to promote the maturation of IL-1 $\beta$ and IL-18 (50). However, GSDMD is dispensable for the release of cytokines from necroptotic cells (51).

\section{APPLICATION OF PYROPTOSIS IN TUMOR TREATMENT}

As inflammatory cell death, the role of pyroptosis in tumor progression and suppression remains elusive. The chronic inflammation caused by inflammatory mediators released from pyroptotic tissues increases the risk of tumorigenesis. HMGB1 released from pyroptotic epithelial cells promotes tumorigenesis of colitis-associated colorectal cancer by activating ERK 1/2 pathway (52). A recent study showed that pyroptosis triggered in the central hypoxic region of tumor provoked tumor progression and correlated with reduced survival (37). However, more researchers have found that some anti-cancer agents can induce pyroptosis to suppress cancers' occurrence and development (25). One possible explanation for the dual effects of pyroptosis is that the chronic inflammation activation facilitates tumor progression, while acute activation of pyroptosis leads to necrotic cell death and represses tumor development (37). As nonapoptotic cell death, pyroptosis can overcome the chemotherapeutic drug resistance associated with apoptosis deficiency. Besides, the combined application of pyroptosis inducers can enhance the therapeutic effect of traditional chemotherapy drugs. Recent studies also found a close relationship between pyroptosis and antitumor immunity. Next, we will systematically summarize the therapeutic role of pyroptosis in different tumors.

\section{Lung Cancer}

GSDMD is highly expressed in non-small cell cancer (NSCLC) and associated with poor prognosis in lung adenocarcinoma. The knockdown of GSDMD restrains NSCLC cell proliferation via intrinsic mitochondrial apoptotic pathways and inhibiting EGFR/ Akt signaling (53). Numerous studies have demonstrated that GSDMD played a key role in the proliferation and death of NSCLC cells. Highly expressed GSDMD also improved the possibility to eliminate tumors through the canonical pyroptosis pathway. In recent years, several substances, including 4hydroxybenacid, Simvastatin, huaier extract, polyphyllin VI, Resibufogenin, have been proved to kill NSCLC via caspase-1dependent pyroptosis (54-58). Polyphyllin VI could elevate ROS/ NF- $\kappa \mathrm{B}$ signaling to activate canonical pyroptosis in A549 and H1299 cell lines. NAC, a ROS scavenger, could reverse Polyphyllin VI-induced pyroptosis accompanied by suppression of NF- $\kappa \mathrm{B}$, NLRP3, and caspase-1 (57). Interestingly, upregulated ROS restrained NF- $\kappa \mathrm{B}$ during pyroptosis induced by Resibufogenin. Thus, the role of $\mathrm{NF}-\mathrm{\kappa B}$ remains elusive. Additionally, Resibufogenin was found to suppress metastasis by caspase-1 activation, which is manifested as downregulated MMP-2, downregulated MMP-9 and upregulated TIMP-3, further strengthening its antitumor effect (58). P53 was also involved in the regulation of NSCLC canonical pyroptosis. Analysis of tumor samples from NSCLC patients found that P53 was positively correlated with pyroptosis at mRNA and protein levels. Overexpression or silencing of P53 in A549 cells elicited or suppressed pyroptosis respectively. The result from immune 
immunoprecipitation also verified the interaction between P53 and NLRP3. Furthermore, P53 was significantly upregulated in LPS-induced A549 pyroptosis. In conclusion, P53 might promote pyroptosis via binding and activating NLRP3 directly (59). Noncoding RNA also participates in the canonical pyroptosis of NSCLC. Bioinformatics analysis showed that LncRNA-XIST was highly expressed in NSCLC and negatively correlated with the average survival of cancer patients. Thus, inhibiting LncRNAXIST may contribute to the treatment of NSCLC patients. Subsequent experiments found that knocking down LncRNAXIST led to pyroptosis by activating the miR-335/SOD2/ROS/ NLRP3 signal pathway (60). In another research, downregulation of LncRNA-XIST was demonstrated to relieve its suppression of Smad2 nuclear translocation, subsequently triggering transcriptional activation of P53 and NLRP3. Therefore, the relationship between XIST and pyroptosis was verified from multiple perspectives (61). In addition to NLRP3/caspase-1/ GSDMD pathway, the induction of GSDME cleavage in NSCLC causes pyroptosis and inhibits proliferation as well. Diverse molecular targeted agents, including trametinib, erlotinib, ceritinib, elicited both apoptosis and secondary pyroptosis of NSCLC. The pyroptosis was achieved through activation of the caspase-3/GSDME signal pathway mediated by mitochondrial intrinsic apoptosis (62). ROS and NF- $\mathrm{BB}$ were also involved in GSDME-dependent pyroptosis. L61H10, a thiopyran derivative, induced an apoptosis-to-pyroptosis switch through inhibiting NF$\kappa B(63)$. NF- $\kappa B$ suppression was also observed during GSDMEdependent pyroptosis triggered by Piperlongumine analogue L50377. The inhibitory effect on NF- $\mathrm{KB}$ was associated with elevated cellular ROS (64). As an inflammatory programmed cell death, pyroptosis also participates in the immunotherapy of NSCLC. In a recent study, researchers designed and constructed a new type of CCCR-modified NK92 cells that overcome the immunosuppressive microenvironment and kill H1299 lung cancer cells through GSDME-dependent pyroptosis. Although the upstream signaling pathway of GSDME activation by NK cells was not well elaborated, the experiment was an interesting attempt to associate pyroptosis with tumor immunity (65). Similar to NK cells, CD8+ T lymphocytes also exhibit the antitumor effect. Compared with unactivated CD8+ T lymphocytes, GSDMD was dramatically upregulated in activated $T$ cells. caspase- $4 / 11$ cleaved GSDMD and enhanced cytolytic capacity of the lymphocytes to promoting the killing effect of H1299 cells. Meanwhile, GSDMD-
NT was found to co-localize with granzyme B in vesicles which polarized toward the immune synapse of T lymphocytes (Table 2). It was speculated that CD8+ T cells might bind to target cells and induce cell death by releasing GSDMD-NT (67).

\section{Digestive System Tumor}

In contrast to normal tissues, caspase-1 is low-expressed in hepatocellular carcinoma (HCC). Relevant studies have shown that berberine, euxanthone, alpinumisoflavone and other drugs could activate caspase-1-dependent pyroptosis and kill HCC cells (68-70). Inhibiting autophagy by chloroquine or ATG5 siRNA enhanced alpinumisoflavone induced pyroptosis (70). Meanwhile, 17 $\beta$-estradiol suppressed autophagy in HCC cells via activating NLRP3/caspase-1 (71). Based on these findings, autophagy was hypothesized to play a protective role in druginduced HCC cell death. In addition to autophagy inhibitors, nanomaterials were also applied to enhance the killing effect of pyroptosis on tumors. A nano-drug delivery system mPEGPLGA-PLL loaded with arsenic trioxide was designed to improve the therapeutic effect of arsenic trioxide on HCC. The results showed that As2O3 and As2O3-NPs induced pyroptosis differently in diverse cell lines. Caspase-3/GSDME was activated in Huh7 and HepG2 cells, while GSDMD was activated in Bel7402 cells. Besides, nanoparticles improved the efficacy of As2O3 by enhancing the endocytosis of tumor cells (72). Sorafenib was found to elicited pyroptosis in tumor-associated macrophages

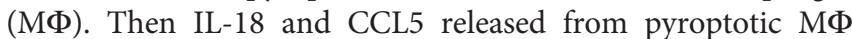
enhanced the chemotaxis and cytotoxicity of NK cells and eliminated HCC via activating antitumor immunity. Blockage of IL-1 $\beta$ or IL-18 could terminate Sorafenib's antitumor effect (73).

The expression levels of caspase-1 and NLRP3 are lower in colorectal cancer (CRC) than adjacent tissues, indicating that pyroptosis associated proteins are negatively correlated with the tumorigenesis and development of CRC. FL118, a camptothecin analog, inhibited proliferation, invasion and metastasis in SW480 and HT129 cells via caspase-1-dependent pyroptosis (74). LXR $\beta$ was found to interact with pannexin one on the cell membrane, causing the release of ATP into the extracellular environment, further activating $\mathrm{P} 2 \times 7$ receptor and NLRP3dependent pyroptosis (75). c9, t11, c15-CLNA (CLNA1), and t9, t11, c15-CLNA (CLNA2) all belong to conjugated $\alpha$-linolenic acid isomers, and both of them showed a potent anti-cancer

TABLE 2 | Summary of pyroptosis introductive treatment in lung cancer.

\begin{tabular}{|c|c|c|c|}
\hline Treatment & Cancer Types & Mechanisms of Pyroptosis Induction & Reference \\
\hline Simvastatin, Huaier extract & NSCLC & NLRP3/Caspase-1/IL-1 $\beta$ \& IL-18 & $(54,56)$ \\
\hline 4-hydroxybenzoic acid & NSCLC & Caspase-1/IL-1 $\beta$ \& IL-18 & $(55)$ \\
\hline Polyphyllin VI & NSCLC & ROS/NF-кB/NLRP3/Caspase-1/GSDMD/IL-1 $\beta$ \& IL-18 & $(57)$ \\
\hline Resibufogenin & NSCLC & ROS/NF- $\kappa B$ suppression/NLRP3/Caspase-1/GSDMD/IL-1 $\beta$ \& IL-18 & (58) \\
\hline LPS & NSCLC & P53/NLRP3/Caspase-1 & (59) \\
\hline LncRNA-XIST Knock-down & NSCLC & miR-335/SOD2/ROS/NLRP3/Caspase-1 & $(60)$ \\
\hline Trametinib, Erlotinib, Ceritinib & NSCLC & BIM/BAX/Cytochrome c/APAF1/Smac/Caspase-9/Caspase-3/GSDME & $(62)$ \\
\hline Paclitaxel, Cisplatin & NSCLC & Caspase-3/GSDME & (66) \\
\hline L61H10 & NSCLC & NF-кB suppression/GSDME & (63) \\
\hline L50377 & NSCLC & ROS/NF- $\kappa B$ suppression/GSDME & (64) \\
\hline CCCR-modified NK92 cells & NSCLC & GSDME & (65) \\
\hline
\end{tabular}


effect through activating canonical and noncanonical pyroptosis respectively (76). LncRNA is also involved in the regulation of CRC pyroptosis. LncRNA RP1-85F18.6 was reported in CRC to promote proliferation and invasion as well as suppress apoptosis and pyroptosis. Knocking down RP1-85F18.6 cleaved GSDMD to trigger pyroptosis. However, the concrete mechanism of GSDMD cleavage was not well elucidated (77). To address the problem of CXCR4+ colorectal cancer stem cells being resistant to multiple chemotherapeutic drugs, the researchers designed a nanostructured toxin T22-DITOX-H6 which contained diphtheria toxin and the CXCR4 ligand T22. The nanostructured toxin induced pyroptosis in colorectal cancer stem cells by binding T22 to CXCR4, specifically manifested by increased NLRP3 and caspase-11 (78). Lobaplatin, a third-generation platinum antineoplastic agent, could remarkably elevate the ROS level in CRC cells and phosphorylate JNK. Then activated JNK caused Baxdependent mitochondrial damage and cytochrome $\mathrm{C}$ release, promoting caspase $3 / 9$ cleavage and GSDME-dependent pyroptosis (79). Redundant ROS was also observed in the process of CRC cell apoptosis and pyroptosis induced by the combination of Arsenic trioxide and Ascorbic acid (80).

In gastric cancer, chemotherapeutic drugs such as cisplatin and 5-Fu were found to induce GSDME-dependent pyroptosis, which could be transformed into apoptosis via GSDME knockout (81, 82). BIX-01294 is a newly discovered autophagy inducer that, when combined with cisplatin, enhances the sensitivity of gastric cancer to cisplatin by activating autophagy (82). In the study of cisplatin resistance, overexpression LncRNA ADAMTS9-AS2 could sponge miR-223-3p to activate NLRP3 and trigger pyroptosis in cisplatinresistant cells (83). Chen et al. found that both Betulinic acid and cisplatin could elicit the cleavage of caspase- 1 in esophageal cancer cells and tissues, and a combination of the two caused more significant cell death (84). PELP1 is a scaffolding oncogenic protein that is closely related to the progression and prognosis of esophageal cancer. Metformin could induce pyroptosis via upregulating miR-497, followed by inhibition of PELP1 to activate the NLRP3/caspase-1/GSDMD pathway (85). Due to the high expression of GSDME in esophageal cancer, some researchers have found that activation of GSDME also led to esophageal cancer pyroptosis. Alpinumisoflavone induced pyroptosis by activating the caspase-3/GSDME pathway. Knocking out of GSDME could convert the pyroptosis into apoptosis (86). PLK1 inhibitor BI2536 could enhance the sensitivity of esophageal squamous cell carcinoma to cisplatin by inhibiting DNA damage repair and inducing pyroptosis. Cisplatin combined with BI2536 triggered esophageal cancer cell death through caspase-3/GSDME pathway, while BI2536 or cisplatin alone did not exhibit morphological changes of pyroptosis (87).

MST1, a vital component of the Hippo pathway, is low expressed in pancreatic cancer. Overexpression of MST1 could increase cellular ROS, activating caspase-1 to elicit pyroptosis. A large number of studies have shown that MST1 played an anti-cancer role in a variety of tumors. Thus, MST1 could be regarded as a potential biomarker and chemotherapy target (88). Gao et al. found that tumor-cellderived microparticles (TMP) could be used as carriers of anticancer drugs to enhance the therapeutic effect. Methotrexate-loaded tumor-cell-derived Microvesicles (MTX-TMPs) perfusion effectively alleviates biliary obstruction caused by extrahepatic cholangiocarcinoma. Further mechanisms studies showed that MTX - TMPs induced pyroptosis of cholangiocarcinoma through the GSDME pathway. Subsequently, the cellular contents released from pyroptosis cells activated macrophages to release inflammatory factors that recruited neutrophils, further enhancing the therapeutic effect of MTX - TMPs perfusion (89).

\section{Central Nervous System Tumor}

Glioblastoma multiforme (GBM) is the most common malignant tumor of the Central Nervous System with a bleak prognosis. Galangin, a natural flavonoid, can induce apoptosis, pyroptosis and protective autophagy in GBM cells. GSDME-NT was significantly elevated in U87MG and U251 cell lines after Galangin treatment. Furthermore, inhibition of autophagy by 3-MA remarkably enhanced apoptosis and pyroptosis triggered by Galangin. Thus, the combined application of autophagy inhibitors and anti-cancer drugs may be helpful for GBM treatment (90). MicroRNAs are also involved in GBM cell pyroptosis. miR-214 can inhibit cell proliferation and migration of U87 and T98G cells through inactivating caspase1. Immunofluorescence analysis of the brain tissue demonstrated that the GBM showed higher levels of caspase-1, IL-1 $\beta$, and IL18 than adjacent tissues, suggesting that caspase- 1 and its substrates could promote GBM proliferation and migration (91).

Another central nervous system tumor associated with pyroptosis is neuroblastoma. Dasatinib-induced neuroblastoma SH-Y5Y cell death exhibited the typical characteristics of pyroptosis. It was accomplished through the activation of caspase- 3 and cleavage of GSDMD and GSDME. Inhibiting caspase- 3 by the specific inhibitor zDEVD notably diminished GSDME-N fragments (Table 3). However, the upstream molecules required for GSDMD cleavage in Dasatinib-induced pyroptosis remained unclear (92).

\section{Melanoma}

Eukaryotic elongation factor-2 kinase (eEF-2K) is a negative regulator of protein synthesis. In GSDME-overexpressed melanoma cells, doxorubicin induced GSDME-dependent pyroptosis and autophagy accompanied by increasing eEf-2K. Silencing eEF-2K could enhance doxorubicin-induced pyroptosis by decreasing autophagy, suggested that doxorubicin induced protective autophagy by activating eEF- $2 \mathrm{~K}$ in melanoma cells during pyroptosis (93). In another study, iron ions were found to raise the ROS level induced by carbonyl cyanide m-chlorophenyl hydrazone (CCCP), thus triggering oxidation and oligomerization of mitochondrial outer membrane protein Tom20. Subsequently, the Bax/caspase/GSDME pathway was activated, leading to pyroptosis of melanoma cells. The study also found that iron co-treatment increased the sensitivity of melanoma to ROS-inducing drugs such as sulfasalazine, buthionine sulfoximine and induced pyroptosis. Therefore, supplementing iron could be helpful for the clinical treatment of melanoma patients (27). The combination of BRAF inhibitors and MEK inhibitors is recommended by the FDA for the treatment of BRAF V600E/K mutant melanoma. Clinical evidence has shown that BRAFi + MEKi co-treatment could induce $\mathrm{T}$ cell 
TABLE 3 | Summary of pyroptosis introductive treatment in digestive system cancer.

\begin{tabular}{|c|c|c|c|}
\hline Treatment & Cancer Types & Mechanisms of Pyroptosis Induction & Reference \\
\hline Berberine & $\mathrm{HCC}$ & Caspase-1 & (68) \\
\hline $\begin{array}{l}\text { Euxanthone, } \\
\text { Alpinumisoflavone, } \\
17 \beta \text {-estradiol }\end{array}$ & $\mathrm{HCC}$ & NLRP3/Caspase- $1 / / L-1 \beta$ \& IL-18 & $(69-71)$ \\
\hline As2O3 & $\mathrm{HCC}$ & $\begin{array}{l}\text { Caspase-3/GSDME (In Huh7 and HepG2) } \\
\text { GSDMD (In Bel-7402) }\end{array}$ & $(72)$ \\
\hline FL118 & Colorectal cancer & NLRP3/Caspase-1/IL-1 $\beta$ \& IL-18 & $(74)$ \\
\hline LXR $\beta$ agonists & Colorectal cancer & LXRß/Pannexin 1/P2×7 receptor/NLRP3/Caspase-1 & (75) \\
\hline $\begin{array}{l}\text { c9, t11, c15-CLNA, } \\
\text { t9, t11, c15-CLNA }\end{array}$ & Colorectal cancer & $\begin{array}{l}\text { Caspase-1/GSDMD (c9, t11, c15-CLNA) } \\
\text { Caspase- } 4 / 5(t 9, \mathrm{t} 11, \mathrm{c} 15-\mathrm{CLNA})\end{array}$ & (76) \\
\hline LncRNA RP1-85F18.6 Knock-down & Colorectal cancer & GSDMD & $(77)$ \\
\hline T22-DITOX-H6 & Colorectal cancer & NLRP3 \& Caspase-11 & (78) \\
\hline Lobaplatin & Colorectal cancer & ROS/JNK/Bax-mitochondrial apoptotic pathway/Caspase-3/9/GSDME & (79) \\
\hline Arsenic trioxide + Ascorbic acid & Colorectal cancer & ROS/Caspase- $1 / \mathrm{IL}-1 \beta$ \& IL-18 & $(80)$ \\
\hline $\begin{array}{l}\text { 5-fluorouracil, } \\
\text { cisplatin }\end{array}$ & Gastric cancer & Caspase-3/GSDME & $(81,82)$ \\
\hline LncRNA ADAMTS9-AS2 & Gastric cancer & LncRNA ADAMTS9-AS2/miR-223-3p/NLRP3/Caspase-1/IL-1 $\beta$ \& IL-18 & (83) \\
\hline $\begin{array}{l}\text { Betulinic acid, } \\
\text { Cisplatin }\end{array}$ & Esophagus cancer & Caspase-1 & (84) \\
\hline Metformin & Esophagus cancer & miR-497/PELP1/NLRP3/Caspase-1/GSDMD & (85) \\
\hline $\begin{array}{l}\text { Alpinumisoflavone } \\
\mathrm{BI} 2536+\text { Cisplatin }\end{array}$ & Esophagus cancer & Caspase-3/GSDME & $(86,87)$ \\
\hline MST1 & Pancreatic Cancer & ROS/Caspase-1/L-1 $\beta$ \& IL-18 & (88) \\
\hline MTX-TMPs & Cholangiocarcinoma & GSDME & (89) \\
\hline
\end{tabular}

infiltration. However, the specific mechanism by which inhibitors induced antitumor immunity is not clear. Erkes et al. found that BRAFi + MEKi combination induced melanoma pyroptosis and release of HMGB1 via activating the caspase-3/GSDME pathway. The released HMGB1 activated dendritic cells to promote inflammatory response, eliciting $\mathrm{T}$ cell activation and antitumor immunity. For BRAFi + MEKi-resistant melanoma, switching to other chemotherapeutic drugs such as etoposide that induces pyroptosis may be an effective strategy (94).

\section{Other Tumors}

In a study of 108 breast cancer and 23 benign lesions adjacent to breast cancer, expression levels of pyroptosis associated proteins were negatively correlated with the pathological grade and TNM staging of breast cancer. Low expressed caspase-1, GSDMD, and IL- $1 \beta$ contributed to proliferation, invasion, and metastasis of breast cancer (95). Omega-3 Docosahexaenoic acid activated caspase-1/GSDMD/IL-1ßpathway to induce pyroptosis in triplenegative breast cancer (96). Wang et al. constructed a bioorthogonal chemical system in which the cancer-imaging probe phenylalanine trifuoroborate (Phe-BF3) specifically identified tumor cells and desilylated and 'cleaved' nanoparticleconjugated gasdermin, promoting the release of GSDMA3 and pyroptosis in breast cancer 4T1 cells. In the tumor-bearing mice model, pyroptosis of less than $15 \%$ of tumor cells led to the elimination of the entire 4T1 mammary tumor graft, which was not observed in immunodeficient mice. Further study showed that pyroptosis induced tumor tissue clearance by activating cytotoxic $\mathrm{T}$ cells and CD4+ T helper cells in the tumor microenvironment. Besides, due to the low inflammation level within the tumor microenvironment, the immune checkpoint blockade (particularly anti-PD1) had a low response rate in mammary tumor treatment. Combination of NP-GSDMA3, Phe-BF3 and
anti-PD1 notably inhibited tumor growth, which indicated that the pyroptosis associated inflammatory response enhanced the antitumor immunity of checkpoint blockade (97). In ovarian cancer, Nobiletin induced increased ROS production and autophagy, promoting cleavage of GSDMD and GSDME (98). 2-(anaphthoyl)ethyltrimethylammonium iodide ( $\alpha$-NETA) induced pyroptosis in ovarian cancer through caspase-4/ GSDMD pathway (99). In cervical cancer, tanshinone II A was found to upregulate caspase-3/9 and GSDMD significantly. Inhibiting miRNA145 reversed tanshinone II A induced pyroptosis (100). Sirt1, highly expressed in cervical cancer, is critical for the development of cervical cancer and is associated with poor clinical outcomes. Knocking down Sirt1 activated AIM2 and caspase- 1 to elicit pyroptosis. Besides, treating healthy cervical cancer cells with culture media from pyroptotic cells also caused AIM2/caspase-1-dependent pyroptosis. Therefore, pyroptotic cervical cancer cells might release certain substances that triggered pyroptosis in adjacent tissues (101). Induction of pyroptosis was also found in studies of endometrial cancer treatment. Pyroptosis associated proteins were highly expressed in endometrial cancer. Yang et al. found that Hydrogen induced pyroptosis in endometrial cancer cells by activating Ros and Mitosox/NLRP3/Caspase-1/GSDMD signaling pathway (102). BRD4, a member of the bromodomain and extra terminal domain (BET) family, can bind to histone tails to change chromatin structure and influence multiple physiological processes. BRD4 inhibitor JQ1 suppressed proliferation and epithelial-mesenchymal transition of renal cancer via triggering pyroptosis (103). Zhang et al. designed and synthesized a series of novel 3',5'-diprenylated chalcones to develop new chemotherapy drugs. One of these chalcones $(\mathrm{C} 10)$ was found to induce both apoptosis and GSDME-dependent pyroptosis through activating $\mathrm{PKC} /$ /JNK signing pathway (38). In nasopharyngeal carcinoma, 
Taxol activated caspase-1/GSDMD pathway to induce pyroptosis. Inhibition of autophagy could improve Taxol's cytotoxicity (104). Protein kinase $\mathrm{R}$ (PKR), a serine-threonine kinase, can be activated by multiple stress signals. Gasticin was found to activate $\mathrm{PKR} / \mathrm{JNK} / \mathrm{NF}-\mathrm{\kappa B} /$ caspase-1 signal pathway to triggering nasopharyngeal carcinoma pyroptosis (105). Besides, lobaplatin was also associated with nasopharyngeal carcinoma pyroptosis, albeit by activating the caspase-3/GSDME pathway. Activating cell inhibitor of apoptosis protein-1/2 (cIAP1/2), inhibiting Ripoptosome (RIPK1/Caspase-8/FADD) or ROS could alleviate lobaplatin induced pyroptosis (106). In osteosarcoma, Dioscin inhibited tumor proliferation by inducing G2/M-phase arrest, apoptosis and GSDME-dependent pyroptosis (107). Anthocyanin induced pyroptosis of oral squamous cancer cells through canonical inflammasome pathway (108). Inflammasome components such as NLRP3 and caspase-1 were low expressed in malignant mesothelioma. Doxorubicin and cisplatin could activate the NLRP3/caspase-1 signal pathway to suppress tumor proliferation, and such pyroptosis was further enhanced with the combination of IL-1 receptor antagonist (109). More drugs inducing pyroptosis in tumors from different tissue sources will be developed for clinical treatment with related research progression (Table 4).

\section{RESEARCH HOTSPOTS IN PYROPTOSIS AND TUMOR THERAPY}

\section{Exploration of the Upstream Pathways That Activate Tumor Pyroptosis}

Caspase and gasdermin family are key molecules in pyroptosis. Exploring the upstream pathways that activate pyroptosis associated molecules contributes to an in-depth understanding of pyroptosis in tumors. ROS has been widely involved in tumor pyroptosis induced by various drugs. On the one hand, ROS activates canonical inflammasome pathway to elicit pyroptosis $(57,60)$. On the other hand, ROS triggers apoptosis and secondary GSDME-dependent pyroptosis $(27,79)$. Activation of NF- $\kappa B$ is intimately related to cancer development. Thus, regulating NF- $\mathrm{KB}$ activity becomes an important strategy for cancer therapy. Resibufogenin, L61H10, JQ1 and other drugs can activate pyroptosis via inhibiting NF- $\mathrm{BB}(58,63,103)$. However, Polyphyllin VI and Casticin were reported to activate NF- $\kappa \mathrm{B}$ and caspase-1-dependent pyroptosis $(57,105)$. Depending on different tumor and drug types, NF- $\kappa \mathrm{B}$ showed both oncogenic and anti-cancer effects. As a transcription factor, NF- $\kappa \mathrm{B}$ increases transcription of several proto-oncogenes, promoting tumor growth and invasion (103). Meanwhile, NF- $\kappa \mathrm{B}$ binds to the NLRP3 promoter, subsequently increasing NLRP3 expression and activating pyroptosis (110). The regulation effect of NF- $\mathrm{KB}$ on pyroptosis needs further investigation. JNK, a member of the MAPK family, responds to various stress signals and plays vital roles in regulating apoptosis and pyroptosis (111). Lobaplatin upregulates JNK to enhance mitochondrial translocation of BAX, activating GSDME-dependent pyroptosis via mitochondrial apoptosis pathway (79). In recent years, more and more researchers focus their attention on the regulatory role of non-coding RNAs in tumorigenesis and development. Some miRNAs and LncRNAs have been identified to be associated with tumor pyroptosis. Generally, miRNAs regulate cell function by binding to the 3'UTR of target mRNA. Depending on the downstream target, specific miRNAs can act as either oncogenes or tumor suppressor genes. In esophageal cancer, miR-497 inhibited PELP1 to activated caspase-1-dependent pyroptosis

TABLE 4 | Summary of pyroptosis introductive treatment in other tumors.

\begin{tabular}{|c|c|c|c|}
\hline Treatment & Cancer Types & Mechanisms of Pyroptosis Induction & Reference \\
\hline Galangin & Glioma & Caspase-3/GSDME & (90) \\
\hline Dasatinib & Neuroblastoma & $\begin{array}{l}\text { Caspase-3/GSDME } \\
\text { GSDMD }\end{array}$ & (92) \\
\hline $\begin{array}{l}\text { Doxoribici, } \\
\text { BRAFi + MEKi, } \\
\text { Etoposide }\end{array}$ & Melanoma & Caspase-3/GSDME & $(93,94)$ \\
\hline Iron + CCCP & Melanoma & ROS/Tom20/Bax/Caspase-3/GSDME & $(27)$ \\
\hline $\mathrm{DHA}$ & Breast cancer & Caspase-1/GSDMD/IL-1 $\beta$ & (96) \\
\hline NP-GSDMA3 + Phe-BF3 & Breast cancer & GSDMA3 & (97) \\
\hline Nobiletin & Ovarian cancer & ROS/Autophagy/GSDMD \& GSDME & (98) \\
\hline$\alpha$-NETA & Ovarian cancer & Caspase-4/GSDMD & (99) \\
\hline Tanshinone II A & Cervical cancer & miR145/GSDMD/IL-1 $\beta$ \& IL-18 & $(100)$ \\
\hline SIRT1 knockdown & Cervical cancer & AlM2/Caspase-1 & $(101)$ \\
\hline Hydrogen & Endometrial cancer & ROS and Mitosox/NLRP3/Caspase-1/GSDMD/IL-1 $\beta$ & $(102)$ \\
\hline 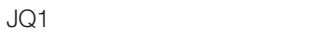 & Renal cancer & NF-кB suppression/NLRP3/Caspase-1/GSDMD & $(103)$ \\
\hline 3',5'-diprenylated chalcones & Prostate cancer & PKCס/JNK/Mitochondrial apoptotic pathway/Caspase-3/GSDME & $(38)$ \\
\hline Taxol & Nasopharyngeal carcinoma & Caspase-1/GSDMD/IL-1 & $(104)$ \\
\hline Casticin & Nasopharyngeal carcinoma & $\mathrm{PKR} / \mathrm{JNK} / \mathrm{NF}-\mathrm{kb} /$ Caspase-1/GSDMD & $(105)$ \\
\hline Lobaplatin & Nasopharyngeal carcinoma & clAP1/2 suppression \& Ripoptosome \& ROS/Caspase-3/GSDME & $(106)$ \\
\hline Dioscin & Osteosarcoma & Caspase-3/GSDME & $(107)$ \\
\hline Anthocyanin & Oral squamous cell carcinoma & NLRP3/Caspase-1/GSDMD/IL-1 $\beta$ & $(108)$ \\
\hline $\begin{array}{l}\text { Doxorubicin, } \\
\text { Cisplatin }\end{array}$ & Mesothelioma & NLRP3/Caspase-1 & $(109)$ \\
\hline
\end{tabular}


(85). In Hela cells, miR-145 upregulated GSDMD, inducing pyroptosis (100). LncRNAs can regulate cell function by targeting miRNAs. Inhibiting LncRNA XIST upregulated miR335 and induced pyroptosis in NSCLC (60). With the continuous development of related research, plenty of molecules such as Sirt, P53, BIM, etc., have been involved in tumor pyroptosis pathways. The exploration of upstream signal molecules of pyroptosis in tumors will help us further understand the role of pyroptosis in tumors and screen new tumor biomarkers and potential therapeutic targets.

\section{Develop New Therapy Based on Pyroptosis}

\section{Screen Effective Chemotherapeutic Drugs}

In general, most clinical chemotherapy drugs inhibit tumor growth, invasion and metastasis via inducing apoptosis. However, in neurofibroma SH-SY5Y or melanoma MeWo cells with high GSDME expression, traditional chemotherapeutic drugs such as doxorubicin, cisplatin can induce pyroptosis through caspase-3/GSDME pathway (25). As mentioned above, more and more chemotherapeutic drugs have been found to trigger pyroptosis through canonical or noncanonical inflammasome pathway. These studies greatly enriched our understanding of the mechanisms of chemotherapeutic drugs and provided a possibility for future drug discovery and development. Natural compounds such as berberine and casticin can activate pyroptosis to inhibit cancer proliferation (68). The use of compounds that are widely available and easy to extract would help reduce the cost of clinical chemotherapy. Besides, the structure of some natural compounds offers prospective possibilities for the synthesis and development of new chemotherapeutic drugs (38). Actively screening and researching chemotherapeutic drugs, natural compounds and artificially synthesized reagents that induce pyroptosis will further improve the existing treatment for malignant tumors.

\section{The Combined Application of Pyroptosis Inductors and Other Anticancer Drugs}

Drug combination has become an important strategy for tumor treatment. The combination of chemotherapeutic drugs with independent mechanisms effectively reduces drug resistance and side effects (112). As a newfound PCD, pyroptosis has shown a bright prospect in combined chemotherapy. In esophageal cancer, betulinic acid elicited caspase-1-dependent pyroptosis. Further study demonstrated that the combination of betulinic acid and cisplatin showed stronger growth inhibition than only one alone (84). As a homeostatic degradative process, autophagy is widely involved in regulating tumor cell proliferation and death. Autophagy inducer BIX-01294 was reported to enhance pyroptosis induced by cisplatin, although the specific mechanisms of autophagy activation were unclear (82). In more cases, however, autophagy plays a role in promoting tumor survival. For instance, the combination of autophagy inhibitor and pyroptosis inductors such as doxorubicin, Taxol significantly suppressed tumor proliferation $(93,104)$.

\section{Pyroptosis and Antitumor Immunity}

As inflammatory cell death, pyroptosis possesses the ability to activate the immune system. Immune stimulants including HMGB1 released from pyroptotic tumor cells can induce the activation of dendritic cells and antitumor T cells. Researchers have found that BRAFi + MEKi treatment in melanoma promoted GSDME-dependent pyroptosis and intensive immune response due to the release of HMGB1 (94). In another study, a biorthogonal system was constructed for selectively releasing active gasdermins into tumor cells. Although the tumor grafts under treatment stopped growth completely, only $10 \%-30 \%$ of the tumor cells underwent pyroptosis. Besides, the tumor regression was abolished in immune-deficient mice (97). All the above results suggested that pyroptosis could play an antitumor role by activating the immune response. On the other hand, tumor-infiltrating immune cells have also been demonstrated to induce pyroptosis of tumor cells. Genetically engineered T cells modified with chimeric antigen receptors (CARs) could induce GSDME-dependent pyroptosis in leukemic cells. What's more, the activated GSDME in leukemic cells was cleaved by granzyme B released from chimeric antigen receptor (CAR) T cells (113). Granzyme A was also proved to induce tumor cell pyroptosis via the cleavage of GSDMB in another recent study (35). The granzyme family seems to play an essential role in pyroptosis triggered by cytotoxic lymphocytes. Furthermore, the activation of pyroptosis pathways in cytotoxic lymphocytes can enhance their cytotoxicity. Researchers have found that GSDMD was upregulated in activated CD8+ T cells and GSDMD deficiency could reduce its cytolytic capacity (67). Immune checkpoint inhibitors (ICIs) have shown a brilliant application prospect in clinical cancer therapy. However, only one-third of patients are responsive to ICIs (114). Recent research showed a synergistic effect between pyroptosis and ICIs. Induction of pyroptosis in target cells sensitized ICI-resistant tumors to checkpoint blockade (97). Due to the cell membrane defect, pyroptotic cells release a large number of cellular contents to induce intense inflammatory responses and massive infiltration of lymphocytes. Markedly increased lymphocyte infiltration further induces caspase-3dependent and independent tumor cell pyroptosis, forming positive feedback to improve the antitumor effect $(89,94,97)$. In conclusion, the bi-directional relationship between pyroptosis and antitumor immunity will be a potential research hotspot.

\section{Pyroptosis and Nanotechnology}

With the progress of material science, nanotechnology has been widely applied in the field of cancer treatment. Nanoparticles can be used as carriers to enhance the antitumor effect of chemotherapeutic drugs. As2O3-NPs enhanced endocytosis of tumor cells to increase the cellular As2 33 content. Then As2O3 exerted antitumor effects via inducing pyroptosis (72). In addition, the application of nanoparticles promotes the distribution of pyroptosis inducers in tumor cells rather than adjacent tissues, which will improve the efficacy and reduce side effects. To address the problem of chemotherapy resistance in CXCR4 overexpressed colon cancer, a nanotoxin composed of the catalytic domain of the diphtheria toxin and CXCR4 ligand T22 was designed. The toxin targeted CXCR4+ 
colon cancer cells specifically and induced pyroptosis (78). In another study, the GSDMA3-NP which could aggregate and release activated GSDMA3 in tumor cells was synthesized for breast cancer treatment (97).

\section{CONCLUSIONS}

Pyroptosis is a newfound inflammatory programmed cell death. Membrane perforation, cell swelling and cell rupture are crucial characteristics of pyroptosis. As inflammatory cell death, pyroptosis is closely associated with tumor growth, proliferation and invasion. Pyroptosis associated proteins such as caspase-1, GSDMD were low expressed in multiple cell types. Therefore, inducing pyroptosis in tumor cells has been a new strategy of cancer treatment. In recent years, various chemotherapy drugs, natural compounds and synthetic medicines were found to induce pyroptosis in different types of cancer cells. Signal molecules such as ROS, NF- $\kappa B$ and non-coding RNAs were widely involved in the activation of pyroptosis. In most cases, pyroptosis is triggered through activating caspase-1/GSDMD or caspase-3/GSDME. Whether the rest members of gasdermin family also induce pyroptosis remains unclear. Studies focused on pyroptosis signaling pathways will provide a new possibility for finding tumor biomarkers and further chemotherapeutic drug development. Apoptosis resistance is the common cause of clinical chemotherapy resistance. Induction of other PCD such as necroptosis, pyroptosis could overcome apoptosis resistance. Thus, the combined application of pyroptosis inductors during chemotherapy will be helpful. Besides, the cellular contents released

\section{REFERENCES}

1. Xia X, Wang X, Cheng Z, Qin W, Lei L, Jiang J, et al. The role of pyroptosis in cancer: pro-cancer or pro-"host"? Cell Death Dis (2019) 10:650. doi: 10.1038/s41419-019-1883-8

2. Galluzzi L, Vitale I, Aaronson SA, Abrams JM, Adam D, Agostinis P, et al. Molecular mechanisms of cell death: recommendations of the Nomenclature Committee on Cell Death 2018. Cell Death Differ (2018) 25:486-541. doi: 10.1038/s41418-017-0012-4

3. Hersh D, Monack DM, Smith MR, Ghori N, Falkow S, Zychlinsky A. The Salmonella invasin SipB induces macrophage apoptosis by binding to caspase-1. Proc Natl Acad Sci U.S.A. (1999) 96:2396-401. doi: 10.1073/ pnas.96.5.2396

4. Brennan MA, Cookson BT. Salmonella induces macrophage death by caspase-1-dependent necrosis. Mol Microbiol (2000) 38:31-40. doi: 10.1046/j.1365-2958.2000.02103.x

5. Cookson BT, Brennan MA. Pro-inflammatory programmed cell death. Trends Microbiol (2001) 9:113-4. doi: 10.1016/s0966-842x(00)01936-3

6. Fink SL, Cookson BT. Caspase-1-dependent pore formation during pyroptosis leads to osmotic lysis of infected host macrophages. Cell Microbiol (2006) 8:1812-25. doi: 10.1111/j.1462-5822.2006.00751.x

7. Fink SL, Cookson BT. Pyroptosis and host cell death responses during Salmonella infection. Cell Microbiol (2007) 9:2562-70. doi: 10.1111/j.14625822.2007.01036.x

8. Shi J, Zhao Y, Wang K, Shi X, Wang Y, Huang H, et al. Cleavage of GSDMD by inflammatory caspases determines pyroptotic cell death. Nature (2015) 526:660-5. doi: 10.1038/nature15514

9. Ding J, Wang K, Liu W, She Y, Sun Q, Shi J, et al. Pore-forming activity and structural autoinhibition of the gasdermin family. Nature (2016) 535:111-6. doi: $10.1038 /$ nature 18590 from pyroptotic tumor cells can promote infiltration of inflammatory cells in the tumor microenvironment and improve antitumor immunity. However, the interaction between pyroptosis and antitumor immunity still needs further research. Nanomaterials are also used in the induction of tumor pyroptosis. Nanomaterials can increase the accumulation of pyroptosis inducers in tumor cells and reduce the cytotoxicity to adjacent tissues, possessing high application value. Some nanoparticles can even trigger pyroptosis directly. In conclusion, induction of pyroptosis holds promise as an effective treatment for malignant tumors.

\section{AUTHOR CONTRIBUTIONS}

LW wrote the manuscript. XQ has put forward many valuable suggestions in the original and revised manuscripts. In addition, he also helped to collect the literature and redraw Figure 1. All authors contributed to the article and approved the submitted version.

\section{FUNDING}

This work was supported by the National Natural Science Foundation of China (No. 81372697, 81701293, 81772669, 81972346), the Changbaishan Scholar Project of Jilin Province (No. 2013026), Scientific Reasearch Foundation of Jilin Province (20190701051GH, 20200201405JC), and the Achievement Transformation Fund of the First Hospital of Jilin University (JDYYZH-1902040).

10. Xia $\mathrm{X}$, Wang $\mathrm{X}$, Zheng $\mathrm{Y}$, Jiang J, Hu J. What role does pyroptosis play in microbial infection? J Cell Physiol (2019) 234:7885-92. doi: 10.1002/jcp.27909

11. Xu YJ, Zheng L, Hu YW, Wang Q. Pyroptosis and its relationship to atherosclerosis. Clin Chim Acta (2018) 476:28-37. doi: 10.1016/j.cca.2017.11.005

12. Wu J, Lin S, Wan B, Velani B, Zhu Y. Pyroptosis in Liver Disease: New Insights into Disease Mechanisms. Aging Dis (2019) 10:1094-108. doi: 10.14336/ad.2019.0116

13. Pirzada RH, Javaid N, Choi S. The Roles of the NLRP3 Inflammasome in Neurodegenerative and Metabolic Diseases and in Relevant Advanced Therapeutic Interventions. Genes (Basel) (2020) 11:131. doi: 10.3390/ genes11020131

14. Zhou CB, Fang JY. The role of pyroptosis in gastrointestinal cancer and immune responses to intestinal microbial infection. Biochim Biophys Acta Rev Cancer (2019) 1872:1-10. doi: 10.1016/j.bbcan.2019.05.001

15. Wang M, Jiang S, Zhang Y, Li P, Wang K. The Multifaceted Roles of Pyroptotic Cell Death Pathways in Cancer. Cancers (Basel) (2019) 11:1313. doi: 10.3390/cancers11091313

16. Zhang Y, Yang H, Sun M, He T, Liu Y, Yang X, et al. Alpinumisoflavone suppresses hepatocellular carcinoma cell growth and metastasis via NLRP3 inflammasome-mediated pyroptosis. Pharmacol Rep (2020) 72:1370-82. doi: 10.1007/s43440-020-00064-8

17. Zheng D, Liwinski T, Elinav E. Inflammasome activation and regulation: toward a better understanding of complex mechanisms. Cell Discovery (2020) 6:36. doi: 10.1038/s41421-020-0167-x

18. Fang Y, Tian S, Pan Y, Li W, Wang Q, Tang Y, et al. Pyroptosis: A new frontier in cancer. BioMed Pharmacother (2020) 121:109595. doi: 10.1016/ j.biopha.2019.109595

19. Mitchell PS, Sandstrom A, Vance RE. The NLRP1 inflammasome: new mechanistic insights and unresolved mysteries. Curr Opin Immunol (2019) 60:37-45. doi: 10.1016/j.coi.2019.04.015 
20. Shi J, Gao W, Shao F. Pyroptosis: Gasdermin-Mediated Programmed Necrotic Cell Death. Trends Biochem Sci (2017) 42:245-54. doi: 10.1016/ j.tibs.2016.10.004

21. Shi J, Zhao Y, Wang Y, Gao W, Ding J, Li P, et al. Inflammatory caspases are innate immune receptors for intracellular LPS. Nature (2014) 514:187-92. doi: $10.1038 /$ nature 13683

22. Kayagaki N, Stowe IB, Lee BL, O'Rourke K, Anderson K, Warming S, et al. Caspase-11 cleaves gasdermin D for non-canonical inflammasome signalling. Nature (2015) 526:666-71. doi: 10.1038/nature15541

23. Kayagaki N, Warming S, Lamkanfi M, Vande Walle L, Louie S, Dong J, et al. Non-canonical inflammasome activation targets caspase-11. Nature (2011) 479:117-21. doi: 10.1038/nature10558

24. Rühl S, Broz P. Caspase-11 activates a canonical NLRP3 inflammasome by promoting K(+) efflux. Eur J Immunol (2015) 45:2927-36. doi: 10.1002/ eji.201545772

25. Wang Y, Gao W, Shi X, Ding J, Liu W, He H, et al. Chemotherapy drugs induce pyroptosis through caspase-3 cleavage of a gasdermin. Nature (2017) 547:99-103. doi: 10.1038/nature22393

26. Rogers C, Fernandes-Alnemri T, Mayes L, Alnemri D, Cingolani G, Alnemri ES. Cleavage of DFNA5 by caspase-3 during apoptosis mediates progression to secondary necrotic/pyroptotic cell death. Nat Commun (2017) 8:14128. doi: $10.1038 /$ ncomms 14128

27. Zhou B, Zhang JY, Liu XS, Chen HZ, Ai YL, Cheng K, et al. Tom20 senses iron-activated ROS signaling to promote melanoma cell pyroptosis. Cell Res (2018) 28:1171-85. doi: 10.1038/s41422-018-0090-y

28. Orning P, Weng D, Starheim K, Ratner D, Best Z, Lee B, et al. Pathogen blockade of TAK1 triggers caspase-8-dependent cleavage of gasdermin D and cell death. Science (2018) 362:1064-9. doi: 10.1126/science.aau2818

29. Sarhan J, Liu BC, Muendlein HI, Li P, Nilson R, Tang AY, et al. Caspase-8 induces cleavage of gasdermin $\mathrm{D}$ to elicit pyroptosis during Yersinia infection. Proc Natl Acad Sci U.S.A. (2018) 115:E10888-e10897. doi: 10.1073/pnas.1809548115

30. Malireddi RKS, Gurung P, Kesavardhana S, Samir P, Burton A, Mummareddy $\mathrm{H}$, et al. Innate immune priming in the absence of TAK1 drives RIPK1 kinase activity-independent pyroptosis, apoptosis, necroptosis, and inflammatory disease. J Exp Med (2020) 217: jem.20191644. doi: 10.1084/jem.20191644

31. Zheng M, Karki R, Vogel P, Kanneganti TD. Caspase-6 Is a Key Regulator of Innate Immunity, Inflammasome Activation, and Host Defense. Cell (2020) 181:674-687.e13. doi: 10.1016/j.cell.2020.03.040

32. Wu D, Chen Y, Sun Y, Gao Q, Yu B, Jiang X, et al. Gasdermin family: a promising therapeutic target for cancers and inflammation-driven diseases. $J$ Cell Commun Signal (2020) 14:293-301. doi: 10.1007/s12079-020-00564-5

33. Kambara H, Liu F, Zhang X, Liu P, Bajrami B, Teng Y, et al. Gasdermin D Exerts Anti-inflammatory Effects by Promoting Neutrophil Death. Cell Rep (2018) 22:2924-36. doi: 10.1016/j.celrep.2018.02.067

34. Panganiban RA, Sun M, Dahlin A, Park HR, Kan M, Himes BE, et al. A functional splice variant associated with decreased asthma risk abolishes the ability of gasdermin B to induce epithelial cell pyroptosis. J Allergy Clin Immunol (2018) 142:1469-1478.e2. doi: 10.1016/j.jaci.2017.11.040

35. Zhou Z, He H, Wang K, Shi X, Wang Y, Su Y, et al. Granzyme A from cytotoxic lymphocytes cleaves GSDMB to trigger pyroptosis in target cells. Science (2020) 368:eaaz7548. doi: 10.1126/science.aaz7548

36. Chen Q, Shi P, Wang Y, Zou D, Wu X, Wang D, et al. GSDMB promotes non-canonical pyroptosis by enhancing caspase-4 activity. J Mol Cell Biol (2019) 11:496-508. doi: 10.1093/jmcb/mjy056

37. Hou J, Zhao R, Xia W, Chang CW, You Y, Hsu JM, et al. PD-L1-mediated gasdermin C expression switches apoptosis to pyroptosis in cancer cells and facilitates tumour necrosis. Nat Cell Biol (2020) 22:1264-75. doi: 10.1038/ s41556-020-0575-Z

38. Zhang Y, Yang J, Wen Z, Chen X, Yu J, Yuan D, et al. A novel 3',5'diprenylated chalcone induces concurrent apoptosis and GSDMEdependent pyroptosis through activating $\mathrm{PKC} / \mathrm{JNK}$ signal in prostate cancer. Aging (Albany NY) (2020) 12:9103-24. doi: 10.18632/aging.103178

39. Naito MG, Xu D, Amin P, Lee J, Wang H, Li W, et al. Sequential activation of necroptosis and apoptosis cooperates to mediate vascular and neural pathology in stroke. Proc Natl Acad Sci U.S.A. (2020) 117:4959-70. doi: $10.1073 /$ pnas. 1916427117
40. Kerr JF, Wyllie AH, Currie AR. Apoptosis: a basic biological phenomenon with wide-ranging implications in tissue kinetics. Br J Cancer (1972) 26:23957. doi: 10.1038/bjc.1972.33

41. Wang H, Sun L, Su L, Rizo J, Liu L, Wang LF, et al. Mixed lineage kinase domain-like protein MLKL causes necrotic membrane disruption upon phosphorylation by RIP3. Mol Cell (2014) 54:133-46. doi: 10.1016/ j.molcel.2014.03.003

42. Laster SM, Wood JG, Gooding LR. Tumor necrosis factor can induce both apoptic and necrotic forms of cell lysis. J Immunol (1988) 141:2629-34.

43. Chen X, He WT, Hu L, Li J, Fang Y, Wang X, et al. Pyroptosis is driven by non-selective gasdermin-D pore and its morphology is different from MLKL channel-mediated necroptosis. Cell Res (2016) 26:1007-20. doi: 10.1038/ cr.2016.100

44. de Vasconcelos NM, Van Opdenbosch N, Van Gorp H, Parthoens E, Lamkanfi M. Single-cell analysis of pyroptosis dynamics reveals conserved GSDMD-mediated subcellular events that precede plasma membrane rupture. Cell Death Differ (2019) 26:146-61. doi: 10.1038/s41418-0180106-7

45. Frank D, Vince JE. Pyroptosis versus necroptosis: similarities, differences, and crosstalk. Cell Death Differ (2019) 26:99-114. doi: 10.1038/s41418-018$0212-6$

46. Zhang Y, Chen X, Gueydan C, Han J. Plasma membrane changes during programmed cell deaths. Cell Res (2018) 28:9-21. doi: 10.1038/cr.2017.133

47. Vandenabeele P, Vandecasteele K, Bachert C, Krysko O, Krysko DV. Immunogenic Apoptotic Cell Death and Anticancer Immunity. Adv Exp Med Biol (2016) 930:133-49. doi: 10.1007/978-3-319-39406-0_6

48. Schwarzer R, Laurien L, Pasparakis M. New insights into the regulation of apoptosis, necroptosis, and pyroptosis by receptor interacting protein kinase 1 and caspase-8. Curr Opin Cell Biol (2020) 63:186-93. doi: 10.1016/ j.ceb.2020.02.004

49. Tsuchiya K, Nakajima S, Hosojima S, Thi Nguyen D, Hattori T, Manh Le T, et al. Caspase-1 initiates apoptosis in the absence of gasdermin D. Nat Commun (2019) 10:2091. doi: 10.1038/s41467-019-09753-2

50. Conos SA, Chen KW, De Nardo D, Hara H, Whitehead L, Núñez G, et al. Active MLKL triggers the NLRP3 inflammasome in a cell-intrinsic manner. Proc Natl Acad Sci U.S.A. (2017) 114:E961-e969. doi: 10.1073/pnas.1613305114

51. Gutierrez KD, Davis MA, Daniels BP, Olsen TM, Ralli-Jain P, Tait SW, et al. MLKL Activation Triggers NLRP3-Mediated Processing and Release of IL$1 \beta$ Independently of Gasdermin-D. J Immunol (2017) 198:2156-64. doi: 10.4049/jimmunol.1601757

52. Tan G, Huang C, Chen J, Zhi F. HMGB1 released from GSDME-mediated pyroptotic epithelial cells participates in the tumorigenesis of colitisassociated colorectal cancer through the ERK1/2 pathway. I Hematol Oncol (2020) 13:149. doi: 10.1186/s13045-020-00985-0

53. Gao J, Qiu X, Xi G, Liu H, Zhang F, Lv T, et al. Downregulation of GSDMD attenuates tumor proliferation via the intrinsic mitochondrial apoptotic pathway and inhibition of EGFR/Akt signaling and predicts a good prognosis in non-small cell lung cancer. Oncol Rep (2018) 40:1971-84. doi: 10.3892/or.2018.6634

54. Wang F, Liu W, Ning J, Wang J, Lang Y, Jin X, et al. Simvastatin Suppresses Proliferation and Migration in Non-small Cell Lung Cancer via Pyroptosis. Int J Biol Sci (2018) 14:406-17. doi: 10.7150/ijbs.23542

55. Sannino F, Sansone C, Galasso C, Kildgaard S, Tedesco P, Fani R, et al. Pseudoalteromonas haloplanktis TAC125 produces 4-hydroxybenzoic acid that induces pyroptosis in human A459 lung adenocarcinoma cells. Sci Rep (2018) 8:1190. doi: 10.1038/s41598-018-19536-2

56. Xie J, Zhuan B, Wang H, Wang Y, Wang X, Yuan Q, et al. Huaier extract suppresses non-small cell lung cancer progression through activating NLRP3-dependent pyroptosis. Anat Rec (Hoboken) (2019) 304:291-301. doi: $10.1002 / a r .24307$

57. Teng JF, Mei QB, Zhou XG, Tang Y, Xiong R, Qiu WQ, et al. Polyphyllin VI Induces Caspase-1-Mediated Pyroptosis via the Induction of ROS/NF- $\mathrm{KB} /$ NLRP3/GSDMD Signal Axis in Non-Small Cell Lung Cancer. Cancers (Basel) (2020) 12:193. doi: 10.3390/cancers12010193

58. Yin H, Liu YG, Li F, Wang LQ, Zha JH, Xia YC, et al. Resibufogenin suppresses growth and metastasis through inducing caspase-1-dependent pyroptosis via ROS-mediated NF- $\mathrm{\kappa B}$ suppression in non-small cell lung cancer. Anat Rec (Hoboken) (2020) 304:302-12. doi: 10.1002/ar.24415 
59. Zhang T, Li Y, Zhu R, Song P, Wei Y, Liang T, et al. Transcription Factor p53 Suppresses Tumor Growth by Prompting Pyroptosis in Non-Small-Cell Lung Cancer. Oxid Med Cell Longev (2019) 2019:8746895. doi: 10.1155/ 2019/8746895

60. Liu J, Yao L, Zhang M, Jiang J, Yang M, Wang Y. Downregulation of LncRNA-XIST inhibited development of non-small cell lung cancer by activating miR-335/SOD2/ROS signal pathway mediated pyroptotic cell death. Aging (Albany NY) (2019) 11:7830-46. doi: 10.18632/aging.102291

61. Xu X, Zhou X, Chen Z, Gao C, Zhao L, Cui Y. Silencing of lncRNA XIST inhibits non-small cell lung cancer growth and promotes chemosensitivity to cisplatin. Aging (Albany NY) (2020) 12:4711-26. doi: 10.18632/aging.102673

62. Lu H, Zhang S, Wu J, Chen M, Cai MC, Fu Y, et al. Molecular Targeted Therapies Elicit Concurrent Apoptotic and GSDME-Dependent Pyroptotic Tumor Cell Death. Clin Cancer Res (2018) 24:6066-77. doi: 10.1158/10780432.Ccr-18-1478

63. Chen L, Weng B, Li H, Wang H, Li Q, Wei X, et al. A thiopyran derivative with low murine toxicity with therapeutic potential on lung cancer acting through a NF- $\kappa \mathrm{B}$ mediated apoptosis-to-pyroptosis switch. Apoptosis (2019) 24:74-82. doi: 10.1007/s10495-018-1499-y

64. Li Q, Chen L, Dong Z, Zhao Y, Deng H, Wu J, et al. Piperlongumine analogue L50377 induces pyroptosis via ROS mediated NF- $\kappa$ B suppression in non-small-cell lung cancer. Chem Biol Interact (2019) 313:108820. doi: 10.1016/j.cbi.2019.108820

65. Lu C, Guo C, Chen H, Zhang H, Zhi L, Lv T, et al. A novel chimeric PD1NKG2D-41BB receptor enhances antitumor activity of NK92 cells against human lung cancer $\mathrm{H} 1299$ cells by triggering pyroptosis. Mol Immunol (2020) 122:200-6. doi: 10.1016/j.molimm.2020.04.016

66. Zhang CC, Li CG, Wang YF, Xu LH, He XH, Zeng QZ, et al. Chemotherapeutic paclitaxel and cisplatin differentially induce pyroptosis in A549 lung cancer cells via caspase-3/GSDME activation. Apoptosis (2019) 24:312-25. doi: 10.1007/s10495-019-01515-1

67. Xi G, Gao J, Wan B, Zhan P, Xu W, Lv T, et al. GSDMD is required for effector CD8(+) T cell responses to lung cancer cells. Int Immunopharmacol (2019) 74:105713. doi: 10.1016/j.intimp.2019.105713

68. Chu Q, Jiang Y, Zhang W, Xu C, Du W, Tuguzbaeva G, et al. Pyroptosis is involved in the pathogenesis of human hepatocellular carcinoma. Oncotarget (2016) 7:84658-65. doi: 10.18632/oncotarget.12384

69. Chen YF, Qi HY, Wu FL. Euxanthone exhibits anti-proliferative and antiinvasive activities in hepatocellular carcinoma by inducing pyroptosis: preliminary results. Eur Rev Med Pharmacol Sci (2018) 22:8186-96. doi: 10.26355/eurrev_201812_16511

70. Clerc P, Jeanjean P, Hallali N, Gougeon M, Pipy B, Carrey J, et al. Targeted Magnetic Intra-Lysosomal Hyperthermia produces lysosomal reactive oxygen species and causes Caspase-1 dependent cell death. J Control Release (2018) 270:120-34. doi: 10.1016/j.jconrel.2017.11.050

71. Wei Q, Zhu R, Zhu J, Zhao R, Li M. E2-Induced Activation of the NLRP3 Inflammasome Triggers Pyroptosis and Inhibits Autophagy in HCC Cells. Oncol Res (2019) 27:827-34. doi: 10.3727/096504018x154629 20753012

72. Hu J, Dong Y, Ding L, Dong Y, Wu Z, Wang W, et al. Local delivery of arsenic trioxide nanoparticles for hepatocellular carcinoma treatment. Signal Transduct Target Ther (2019) 4:28. doi: 10.1038/s41392-019-0062-9

73. Hage C, Hoves S, Strauss L, Bissinger S, Prinz Y, Pöschinger T, et al. Sorafenib Induces Pyroptosis in Macrophages and Triggers Natural Killer Cell-Mediated Cytotoxicity Against Hepatocellular Carcinoma. Hepatology (2019) 70:1280-97. doi: 10.1002/hep.30666

74. Tang Z, Ji L, Han M, Xie J, Zhong F, Zhang X, et al. Pyroptosis is involved in the inhibitory effect of FL118 on growth and metastasis in colorectal cancer. Life Sci (2020) 257:118065. doi: 10.1016/j.lfs.2020.118065

75. Derangère V, Chevriaux A, Courtaut F, Bruchard M, Berger H, Chalmin F, et al. Liver $\mathrm{X}$ receptor $\beta$ activation induces pyroptosis of human and murine colon cancer cells. Cell Death Differ (2014) 21:1914-24. doi: 10.1038/ cdd.2014.117

76. Ren Q, Yang B, Zhu G, Wang S, Fu C, Zhang H, et al. Antiproliferation Activity and Mechanism of c9, t11, c15-CLNA and t9, t11, c15-CLNA from Lactobacillus plantarum ZS2058 on Colon Cancer Cells. Molecules (2020) 25:1225. doi: 10.3390/molecules25051225
77. Ma Y, Chen Y, Lin C, Hu G. Biological functions and clinical significance of the newly identified long non-coding RNA RP1-85F18.6 in colorectal cancer. Oncol Rep (2018) 40:2648-58. doi: 10.3892/or.2018.6694

78. Serna N, Álamo P, Ramesh P, Vinokurova D, Sánchez-García L, Unzueta U, et al. Nanostructured toxins for the selective destruction of drug-resistant human CXCR4(+) colorectal cancer stem cells. J Control Release (2020) 320:96-104. doi: 10.1016/j.jconrel.2020.01.019

79. Yu J, Li S, Qi J, Chen Z, Wu Y, Guo J, et al. Cleavage of GSDME by caspase-3 determines lobaplatin-induced pyroptosis in colon cancer cells. Cell Death Dis (2019) 10:193. doi: 10.1038/s41419-019-1441-4

80. Tian W, Wang Z, Tang NN, Li JT, Liu Y, Chu WF, et al. Ascorbic Acid Sensitizes Colorectal Carcinoma to the Cytotoxicity of Arsenic Trioxide via Promoting Reactive Oxygen Species-Dependent Apoptosis and Pyroptosis. Front Pharmacol (2020) 11:123:123. doi: 10.3389/fphar.2020.00123

81. Wang Y, Yin B, Li D, Wang G, Han X, Sun X. GSDME mediates caspase-3dependent pyroptosis in gastric cancer. Biochem Biophys Res Commun (2018) 495:1418-25. doi: 10.1016/j.bbrc.2017.11.156

82. Deng BB, Jiao BP, Liu YJ, Li YR, Wang GJ. BIX-01294 enhanced chemotherapy effect in gastric cancer by inducing GSDME-mediated pyroptosis. Cell Biol Int (2020) 44:1890-99. doi: 10.1002/cbin.11395

83. Ren N, Jiang T, Wang C, Xie S, Xing Y, Piao D, et al. LncRNA ADAMTS9AS2 inhibits gastric cancer (GC) development and sensitizes chemoresistant GC cells to cisplatin by regulating miR-223-3p/NLRP3 axis. Aging (Albany NY) (2020) 12:11025-41. doi: 10.18632/aging.103314

84. Chen J, Peng R, Niu Z, Zhou H, Kang C. Betulinic acid enhanced the chemical sensitivity of esophageal cancer cells to cisplatin by inducing cell pyroptosis and reducing cell stemness. Ann Palliat Med (2020) 9:1912-20. doi: 10.21037/apm-20-867

85. Wang L, Li K, Lin X, Yao Z, Wang S, Xiong X, et al. Metformin induces human esophageal carcinoma cell pyroptosis by targeting the miR-497/ PELP1 axis. Cancer Lett (2019) 450:22-31. doi: 10.1016/j.canlet.2019.02.014

86. Zhang B, Zhu WY, Tian H, Zhang HR. Alpinumisoflavone triggers GSDMEdependent pyroptosis in esophageal squamous cell carcinomas. Anat Rec (Hoboken) (2020) 304:323-32. doi: 10.1002/ar.24414

87. Wu M, Wang Y, Yang D, Gong Y, Rao F, Liu R, et al. A PLK1 kinase inhibitor enhances the chemosensitivity of cisplatin by inducing pyroptosis in oesophageal squamous cell carcinoma. EBioMedicine (2019) 41:244-55. doi: 10.1016/j.ebiom.2019.02.012

88. Cui J, Zhou Z, Yang H, Jiao F, Li N, Gao Y, et al. MST1 Suppresses Pancreatic Cancer Progression via ROS-Induced Pyroptosis. Mol Cancer Res (2019) 17:1316-25. doi: 10.1158/1541-7786.Mcr-18-0910

89. Gao Y, Zhang H, Zhou N, Xu P, Wang J, Gao Y, et al. Methotrexate-loaded tumour-cell-derived microvesicles can relieve biliary obstruction in patients with extrahepatic cholangiocarcinoma. Nat BioMed Eng (2020) 4:743-53. doi: 10.1038/s41551-020-0583-0

90. Kong Y, Feng Z, Chen A, Qi Q, Han M, Wang S, et al. The Natural Flavonoid Galangin Elicits Apoptosis, Pyroptosis, and Autophagy in Glioblastoma. Front Oncol (2019) 9:942:942. doi: 10.3389/fonc.2019.00942

91. Jiang Z, Yao L, Ma H, Xu P, Li Z, Guo M, et al. miRNA-214 Inhibits Cellular Proliferation and Migration in Glioma Cells Targeting Caspase 1 Involved in Pyroptosis. Oncol Res (2017) 25:1009-19. doi: 10.3727/096504016x1 4813859905646

92. Zhang J, Chen Y, He Q. Distinct characteristics of dasatinib-induced pyroptosis in gasdermin E-expressing human lung cancer A549 cells and neuroblastoma SH-SY5Y cells. Oncol Lett (2020) 20:145-54. doi: 10.3892/ ol.2020.11556

93. Yu P, Wang HY, Tian M, Li AX, Chen XS, Wang XL, et al. Eukaryotic elongation factor-2 kinase regulates the cross-talk between autophagy and pyroptosis in doxorubicin-treated human melanoma cells in vitro. Acta Pharmacol Sin (2019) 40:1237-44. doi: 10.1038/s41401-019-0222-z

94. Erkes DA, Cai W, Sanchez IM, Purwin TJ, Rogers C, Field CO, et al. Mutant BRAF and MEK Inhibitors Regulate the Tumor Immune Microenvironment via Pyroptosis. Cancer Discovery (2020) 10:254-69. doi: 10.1158/21598290.Cd-19-0672

95. Wu X, Mao X, Huang Y, Zhu Q, Guan J, Wu L. Detection of proteins associated with the pyroptosis signaling pathway in breast cancer tissues and their significance. Int J Clin Exp Pathol (2020) 13:1408-14. 
96. Pizato N, Luzete BC, Kiffer L, Corrêa LH, de Oliveira Santos I, Assumpção JAF, et al. Omega-3 docosahexaenoic acid induces pyroptosis cell death in triple-negative breast cancer cells. Sci Rep (2018) 8:1952. doi: 10.1038/ s41598-018-20422-0

97. Wang Q, Wang Y, Ding J, Wang C, Zhou X, Gao W, et al. A bioorthogonal system reveals antitumour immune function of pyroptosis. Nature (2020) 579:421-6. doi: 10.1038/s41586-020-2079-1

98. Zhang R, Chen J, Mao L, Guo Y, Hao Y, Deng Y, et al. Nobiletin Triggers Reactive Oxygen Species-Mediated Pyroptosis through Regulating Autophagy in Ovarian Cancer Cells. J Agric Food Chem (2020) 68:132636. doi: 10.1021/acs.jafc.9b07908

99. Qiao L, Wu X, Zhang J, Liu L, Sui X, Zhang R, et al. $\alpha$-NETA induces pyroptosis of epithelial ovarian cancer cells through the GSDMD/caspase-4 pathway. FASEB J (2019) 33:12760-7. doi: 10.1096/fj.201900483RR

100. Tong W, Guo J, Yang C. Tanshinone II A enhances pyroptosis and represses cell proliferation of HeLa cells by regulating miR-145/GSDMD signaling pathway. Biosci Rep (2020) 40:BSR20200259. doi: 10.1042/bsr20200259

101. So D, Shin HW, Kim J, Lee M, Myeong J, Chun YS, et al. Cervical cancer is addicted to SIRT1 disarming the AIM2 antiviral defense. Oncogene (2018) 37:5191-204. doi: 10.1038/s41388-018-0339-4

102. Yang Y, Liu PY, Bao W, Chen SJ, Wu FS, Zhu PY. Hydrogen inhibits endometrial cancer growth via a ROS/NLRP3/caspase-1/GSDMD-mediated pyroptotic pathway. BMC Cancer (2020) 20:28. doi: 10.1186/s12885-019-6491-6

103. Tan YF, Wang M, Chen ZY, Wang L, Liu XH. Inhibition of BRD4 prevents proliferation and epithelial-mesenchymal transition in renal cell carcinoma via NLRP3 inflammasome-induced pyroptosis. Cell Death Dis (2020) 11:239. doi: 10.1038/s41419-020-2431-2

104. Wang X, Li H, Li W, Xie J, Wang F, Peng X, et al. The role of Caspase-1/ GSDMD-mediated pyroptosis in Taxol-induced cell death and a Taxolresistant phenotype in nasopharyngeal carcinoma regulated by autophagy. Cell Biol Toxicol (2020) 36:437-57. doi: 10.1007/s10565-020-09514-8

105. Jiang C, Shi R, Chen B, Yan X, Tang G. Casticin elicits inflammasomeinduced pyroptosis through activating $\mathrm{PKR} / \mathrm{JNK} / \mathrm{NF}-\kappa \mathrm{B}$ signal in $5-8 \mathrm{~F}$ cells. BioMed Pharmacother (2020) 123:109576. doi: 10.1016/j.biopha.2019. 109576

106. Chen Z, Xu G, Wu D, Wu S, Gong L, Li Z, et al. Lobaplatin induces pyroptosis through regulating cIAP1/2, Ripoptosome and ROS in nasopharyngeal carcinoma. Biochem Pharmacol (2020) 177:114023. doi: $10.1016 /$ j.bcp.2020.114023
107. Ding Q, Zhang W, Cheng C, Mo F, Chen L, Peng G, et al. Dioscin inhibits the growth of human osteosarcoma by inducing G2/M-phase arrest, apoptosis, and GSDME-dependent cell death in vitro and in vivo. J Cell Physiol (2020) 235:2911-24. doi: 10.1002/jcp.29197

108. Yue E, Tuguzbaeva G, Chen X, Qin Y, Li A, Sun X, et al. Anthocyanin is involved in the activation of pyroptosis in oral squamous cell carcinoma. Phytomedicine (2019) 56:286-94. doi: 10.1016/j.phymed.2018.09.223

109. Westbom C, Thompson JK, Leggett A, MacPherson M, Beuschel S, Pass H, et al. Inflammasome Modulation by Chemotherapeutics in Malignant Mesothelioma. PloS One (2015) 10:e0145404. doi: 10.1371/ journal.pone.0145404

110. Qiao Y, Wang P, Qi J, Zhang L, Gao C. TLR-induced NF- $\kappa B$ activation regulates NLRP3 expression in murine macrophages. FEBS Lett (2012) 586:1022-6. doi: 10.1016/j.febslet.2012.02.045

111. Sui X, Kong N, Ye L, Han W, Zhou J, Zhang Q, et al. p38 and JNK MAPK pathways control the balance of apoptosis and autophagy in response to chemotherapeutic agents. Cancer Lett (2014) 344:174-9. doi: 10.1016/ j.canlet.2013.11.019

112. Pritchard JR, Lauffenburger DA, Hemann MT. Understanding resistance to combination chemotherapy. Drug Resist Update (2012) 15:249-57. doi: 10.1016/j.drup.2012.10.003

113. Liu Y, Fang Y, Chen X, Wang Z, Liang X, Zhang T, et al. Gasdermin Emediated target cell pyroptosis by CAR $\mathrm{T}$ cells triggers cytokine release syndrome. Sci Immunol (2020) 5:eaax7969. doi: 10.1126/sciimmunol. aax7969

114. Jiang P, Gu S, Pan D, Fu J, Sahu A, Hu X, et al. Signatures of T cell dysfunction and exclusion predict cancer immunotherapy response. Nat Med (2018) 24:1550-8. doi: 10.1038/s41591-018-0136-1

Conflict of Interest: The authors declare that the research was conducted in the absence of any commercial or financial relationships that could be construed as a potential conflict of interest.

Copyright (c) 2021 Wang, Qin, Liang and Ge. This is an open-access article distributed under the terms of the Creative Commons Attribution License (CC BY). The use, distribution or reproduction in other forums is permitted, provided the original author(s) and the copyright owner(s) are credited and that the original publication in this journal is cited, in accordance with accepted academic practice. No use, distribution or reproduction is permitted which does not comply with these terms. 